\title{
Internet of Things for improving Supply Chain Performance: A Qualitative Study of Australian Retailers
}

\author{
Tharaka de Vass \\ College of Business \\ Victoria University \\ Melbourne, Australia \\ Tharaka.DeVass@vu.edu.au
}

\author{
Himanshu Shee \\ College of Business \\ Victoria University \\ Melbourne, Australia \\ Himanshu.Shee@vu.edu.au
}

\author{
Shah Jahan Miah \\ College of Business \\ Victoria University \\ Melbourne, Australia \\ Shah.Miah@vu.edu.au
}

\begin{abstract}
Internet of Things (IoT) is a global platform of Internet connected smart devices that have been argued to improve the supply chain integration (SCI). While the positive effect of IoT capability on SCI influencing supply chain and firm performance is empirically validated, exploratory study in this context is limited. Drawing upon the organisational capability theory, this study investigates the use of IoT in enhancing the integration of suppliers, customers and internal activities of the retail supply chain. Interviews of managers across Australian retail industry were analysed using Nvivo. The thematic analysis reveals the added capability of IoT that improves supply chain visibility, auto-capture and information sharing for greater SCI. The IoT-enabled integration capability demonstrates a positive effect on cost, quality, delivery, and flexibility of the entire supply chain and improves retail firm's sustainable performance with financial, social and environmental outcomes.
\end{abstract}

Keywords Internet of Things (IoT), supply chain integration, supply chain performance, firm performance, organisational capability theory 


\section{Introduction}

Internet of Things (IoT) is defined as a global platform of Internet connected smart objects that allows things to connect anytime, anywhere using any network or service (Atzori, Iera, \& Morabito, 2010; Borgia, 2014; de Vass, Shee \& Miah, 2018). It is viewed as an extension of legacy ICT systems (e.g. computers, ERP, email, fax, phone, and WMS) that traditionally facilitate information sharing among individuals, organisations, and industries (Borgia, 2014). Consequently, IoT act as emerging information service architecture facilitating the exchange of goods in global supply networks (Liu \& Sun, 2011). The RFID tags, sensors, actuators, mobile phones and GPS services are reported to have the capability of conducting real-time data capture and monitoring of almost every link in contemporary supply chains (Ben-Daya, Hassini, \& Bahroun, 2017).

In an era where supply chain performance is presumed as key enabler of firms' sustainability, supply chain integration (SCI) mechanism has emerged as a key driver in improving such performance (AlfallaLuque, Medina-Lopez, \&Dey, 2013). SCI is defined as collaborative internal and external management of strategic, tactical and operational business processes to achieve effective and efficient flows of products, information and funds to provide the maximum value to the end customer at the lowest cost and the greatest speed (Alfalla-Luque et al., 2013; Huo, 2012; Yu, 2015). Within the context, ICT is traditionally considered as a digital enabler of SCI, by facilitating inter- and intra-firm information flows (Rai, Patnayakuni, \& Seth, 2006). Consequently, multitude of studies confirm the positive effect of ICT on SCI (Kim, 2017; Li, Yang, Sun, \& Sohal, 2009; Rai et al., 2006).

However, the limitation of traditional ICT in sensing and capturing additional transactional data has paved the way to emerging services and applications such as IoT that facilitate interconnection of physical things with digital world (Constantinides, Kahlert, \& de Vries, 2017; de Vass et al., 2018). Further, it offers a safe and trustworthy platform to exchange information related to goods and services in a global supply chain (Mishra et al., 2016). However, there is limited empirical evidence on its effect on supply chain and firm performance (Mishra et al., 2016), except the recent study done by de Vass, Shee, \&Miah (2018).

Research on IoT is still emerging (Liu \& Gao, 2014), and its potential benefits are seldom explored (Whitmore, Agarwal, \&Da Xu, 2014). Despite conceptualisations (Ping, Liu, Zhou, and Wang, 2011) and laboratory experimentation (Yan et al., 2014), the use of IoT is still in its early stages for some retailers. Others are claiming to have IoT in some form or other for sometimes now. Further, literature calls for empirical evidence of IoT effect on SCI. To fill this knowledge gap, de Vass et al. (2018)'s survey-based empirical framework confirms the positive effect of IoT capability on three dimensions (i.e., supplier, internal and supplier integration) of SCI impacting supply chain and firm performance, from the Australian retail industry perspective. However, the study lacks descriptive insights to explain the ground reality of IoT in use.

Retail is an industry reported to be at the forefront in embracing IoT (Balaji \& Roy, 2017), as it faces various challenges due to its dynamic nature and unexpected consumer expectation (Majeed \& Rupasinghe, 2017). IoT use provides innovative approaches for retailers (Constantinides et al., 2017). From organisational capability perspective, IoT adoption can be viewed as an additional capability that can add to the current configuration of ICT capability for greater SCI (de Vass et al., 2018). However, the current state of IoT use is quite rhetoric and fragmented in discussion among practitioners and academics.

Hence, the aim of the qualitative study is to investigate the ground reality of IoT use in Australian retailers to drive their supply chain performance. The sub-objectives are: (i) to find out the extent IoT help in supplier process integration; (ii) the extent IoT help in customer process integration; (iii) the extent IoT help in internal integration, and (iv) extent it helps in performance improvement of supply chain firms. The research question developed to achieve these objectives is how IoT technologies drive supply chain processes with suppliers, customers and internal activities of the retail firms.

This paper is set out as follows. First, the literature on SCI, ICT and IoT is reviewed. The methodology of data collection via semi-structured interviews and analysis techniques is then outlined. Discussion, theoretical and managerial implications are presented. Finally, the study concludes with a summary and limitations of the study. 


\section{Literature background}

\subsection{Supply chain integration and performance}

SCI is conceptualised as a significant contributor to supply chain performance (Alfalla-Luque et al., 2013; Huo, 2012). Although some scholars examined SCI as a single construct, multi-dimensional (e.g., internal, supplier and customer integration) approach has been the preferred alternative (Alfalla-Luque et al., 2013). Internal integration can break down cross-functional barriers and facilitate real-time information sharing across key functions; supplier integration involves collaboration between a focal firm and its suppliers in managing cross-firm business processes; customer integration enables a deeper understanding of market expectations and opportunities in response to customer requirements (Huo, 2012; Yu, 2015). These internal and external integrating capabilities directly and indirectly contribute to performance (Huo, 2012). Within this context, ICT is considered as a digital enabler for SCI, via enhanced information flows (Li et al., 2009; Rai et al., 2006).

\subsection{Information and Communication Technologies and Supply Chain Integration}

Ample studies confirm ICT as an enabler of SCI for performance gain (Kim, 2017; Li et al., 2009; Rai et al., 2006). The findings are consistent with organisational capability theoretical perspective, where ICT is considered as having an impact on performance through a higher-order capability, in this case integration (Rai et al., 2006). The conceptualisation is that ICT implementation itself cannot generate performance; its business alignment and application to facilitate SCI is what renders positive outcomes (Kim, 2017; Li et al., 2009; Rai et al., 2006). Incidentally, the SCI studies have been predominantly survey-based, conducted on legacy ICT systems (Kim, 2017; Li et al., 2009; Rai et al., 2006).

\subsection{Internet of Things and Supply Chain Integration}

The emerging IoT is expected to play a leading role in the Industry 4.0 era, where the integration of IoT into logistic digitalisation is becoming more and more relevant (Hofmann \& Rüsch, 2017). Thus, IoT is perceived to facilitate a technological shift in supply chain management (Ben-Daya et al., 2017). Supply chain information systems based on IoT are capable to coordinate and integrate internal and external activities of enterprises (Cui, 2015). Correspondingly, SCI is reported to be greatly enhanced with the proliferation of IoT (Haddud, DeSouza, Khare, \& Lee, 2017).

Scholars have acknowledged the effect of IoT on SCI in their studies (Cui, 2015; Haddud et al., 2017). Ping et al. (2011)'s conceptualisation provides a detailed account on how IoT bridges the gap between physical objects and digital world, to establish a connection between the physical flow and the information flow. However, among the scant literature that validates IoT SCI attributes, Yan et al. (2014)'s study tested the relationship between IoT and SCI, in controlled laboratory conditions to confirm its integration capability. Later, de Vass et al. (2018) offer empirical evidence on IoT capability having a significant effect on internal, customer and supplier integration processes to impact supply chain and firm sustainable performance. However, despite surveys grant statistical generalisation, it has been criticised for over-simplification of reality (Wieland \& Marcus Wallenburg, 2012). Since, empirical researchers primarily utilise quantitative approaches, demand is however rising for qualitative perspectives (Wieland \& Marcus Wallenburg, 2012). In consequence, interview based qualitative studies on this theme is yet to be undertaken, a gap that this research seeks to address.

\section{Study methodology}

This study is exploratory in nature, thus a qualitative inquiry was conducted on firms representing a cross section of retail industry, to draw critical process information. The respondents possessed technology enabled supply chain management knowledge and represented various retail subdivisions or sectors (ABS ANZSIC, 2013). The interviewees' profiles are displayed in Table 1 . The majority of the participant retailers (7) were large ( $>200$ employees) and the rest (5) were medium $(20>\&<200)$ and represented all retail subdivisions (ABS_ANZSIC, 2013).

Table 1: Summary profile of the interviewees (retailers)

\begin{tabular}{|l|l|l|l|l|l|l|l|}
\hline ID & $\begin{array}{l}\text { Cod } \\
\text { e }\end{array}$ & $\begin{array}{l}\text { Work } \\
\text { exp. } \\
\text { (Yrs.) }\end{array}$ & J ob role & Retail sector & Key retail form & Firm size & $\begin{array}{l}\text { First } \\
\text { adopted IoT }\end{array}$ \\
\hline 1 & A & 2 & $\begin{array}{l}\text { Supply chain } \\
\text { manager }\end{array}$ & Cosmetic and toiletry & Multi-model & Medium & $\begin{array}{l}\text { Less than 2 } \\
\text { years ago }\end{array}$ \\
\hline
\end{tabular}




\begin{tabular}{|l|l|l|l|l|l|l|l|}
\hline 2 & B & 11 & $\begin{array}{l}\text { Supply chain } \\
\text { manager }\end{array}$ & Department store & $\begin{array}{l}\text { Bricks and } \\
\text { mortar }\end{array}$ & Large & $\begin{array}{l}\text { Over 11 } \\
\text { years }\end{array}$ \\
\hline 3 & C & 3 & $\begin{array}{l}\text { Supply chain } \\
\text { manager }\end{array}$ & Supermarket & $\begin{array}{l}\text { Bricks and } \\
\text { mortar }\end{array}$ & Large & 4 years now \\
\hline 4 & D & 2 & $\begin{array}{l}\text { Supply chain } \\
\text { manager }\end{array}$ & Pet products & Multi-model & Large & $\begin{array}{l}5 \text { years at- } \\
\text { least }\end{array}$ \\
\hline 5 & E & 3 & Owner & $\begin{array}{l}\text { Restaurant/ café/ take- } \\
\text { away }\end{array}$ & Multi-model & Medium & 3 years now \\
\hline 6 & F & 4 & $\begin{array}{l}\text { Supply chain } \\
\text { manager }\end{array}$ & $\begin{array}{l}\text { Telecommunication } \\
\text { products / Electronics }\end{array}$ & Multi-model & Large & $\begin{array}{l}\text { 3 years at } \\
\text { least }\end{array}$ \\
\hline 7 & G & 5 & $\begin{array}{l}\text { Supply chain } \\
\text { manager }\end{array}$ & $\begin{array}{l}\text { Clothing, footwear } \\
\text { and personal } \\
\text { accessories }\end{array}$ & Multi-model & Large & $\begin{array}{l}\text { Over 15 } \\
\text { years }\end{array}$ \\
\hline 9 & I & 5 & $\begin{array}{l}\text { Supply chain } \\
\text { manager }\end{array}$ & $\begin{array}{l}\text { Supermarket } \\
\text { and Electronics }\end{array}$ & $\begin{array}{l}\text { Bricks and } \\
\text { mortar }\end{array}$ & Large & $\begin{array}{l}\text { Must years at- } \\
\text { least }\end{array}$ \\
\hline 10 & J & 20 & Store manager & $\begin{array}{l}\text { Fuel and convenience } \\
\text { stores }\end{array}$ & $\begin{array}{l}\text { Bricks and } \\
\text { mortar }\end{array}$ & Large & 5 years now \\
\hline 11 & K & 5 & $\begin{array}{l}\text { IT manager } \\
\text { least }\end{array}$ & $\begin{array}{l}\text { Security and } \\
\text { surveillance/ } \\
\text { Electronics }\end{array}$ & Multi-model & Medium & 5years now \\
\hline 12 & L & 7 & $\begin{array}{l}\text { General } \\
\text { manager }\end{array}$ & Household goods & E-tail & Medium & 6 years now \\
\hline
\end{tabular}

One-on-one semi-structured interviews were conducted with the selected volunteers following de Vass et al. (2018)'s survey study. Semi-structured interviews helped gain rich insights into the core themes. The selection was mainly based on the retail sector, firm size, the retail form and the level of IoT deployment in their respective supply chain operations. The interview schedule had 15 questions under two sections. Section 1 was to understand the firm and the respondent demographics. Section 2 reflected on IoT deployment in in-house, supplier and customer related operations and their respective performance outcomes. IoT-enabled supply chain performance was probed under traditional cost, quality, delivery, flexibility dimensions and firm performance was explored under prevailing triplebottom-lines (i.e., economic, environmental and social) of sustainability. The interviews lasted 45 minutes to 1 hour for each case, and were audio recorded. Following the transcription of the 12 interviews, they were analysed using a typical open-coding process used in qualitativeresearch (Creswell \& Poth 2017). Nvivo 11 was used for thematic analysis. A data reduction process was conducted, using an inductive approach to transform data into orderly and simplified themes to develop a meaningful outcome. This process involved reducing and categorising the text into meaningful segments and labelling them with an appropriate title to best define the material (Creswell \& Poth 2017).

\section{Study findings}

All managers had the understanding of IoT and its inherent potential that were harmonised with academic conceptualisations.

"It is an umbrella term used universally for the mechanism behind it. IoT are the devices that capitalise the power of Internet" [Retailer I].

All believed that they had IoT in place to a reasonable degree, as a mix of 'things' across the supply chain in different operational processes with different intensity. Many referred to retail industry as 'very competitive' and IoT as technologies can help them gain a competitive edge. All commended on IoT potential in supply chain operations.

\subsection{IoT technologies in Australian retail supply chain}

Many technologies that narrated under IoT umbrella currently coexist, providing added digital capabilities to supply chain retailers. RFID, one form of IoT, at unit-level (e.g., pallet, container) in warehousing and transportation is evident. Barcode scanners, PDA's (personal digital assistant), RF (radio frequency) scanners, Laser, LED scanners and camera-based scanners are widespread in practice.

In warehousing, hand-held devices (e.g., PDA's, RF scanners) would provide a picking order, conform the product picking by scanning. Also, pick-to-voice, Automatic guided vehicles (AGV), and automatic pallet mover or conveyor control systems are used in operations. In retail store environment, handheld sensors and devices, POS devices, video analytics, IP Cameras, barcoding (unique for some perishable 
items) and mobile payments, including Apple Pay, are already in use. In transport, IoT-enabled (i.e. sensor) track and trace systems, fleet controlling devices, vehicle tracking, and route optimisation are ongoing. IoT-enabled sensor networks in cold-chain logistics track and trace temperature-sensitive products. In food industry, customer can log into a portal on their smartphone to order, pay and track deliverers (e.g., Uber eats).

\subsection{How IoT enables supplier integration}

IoT applications in upstream logistics processes were positively conversed by all.

"I definitely believe that these devices play a big role by bringing us and our suppliers together. Better connection between the partners will only improve the activities and its processes" [Retailer E].

The major themes were around suppliers' operational improvement, communication, forecasting, inbound delivery, receiving and traceability.

The theme of how IoT can help suppliers fulfil retailers' requirement, was discussed by ten retailers. The retailers described many IoT forms at the supplier end to improve their own processes while helping the retailers. The hand-held devices, scanners, labellers, QR codes, barcodes, NFC (near field communicators), smart phones, tablets, various sensors in manufacturing and warehousing environments, image recognition, scan picking, pick-ti-voice picking were some of the common IoT forms. Retailer F stated that IoT helped their manufacturers better understand their processes by evaluating performance of the machines, energy consumption, ambient conditions, status of inventory, the flow of materials via hand-held devices, sensors and RFID.

IoT is important in strengthening communication with suppliers in stock ordering and other upstream information exchanges, according to eight retailers. Apps, Tabs, portals, and hand-held devices in supplier dispatch with real-time updates are very common. Eight retailers discussed forecasting process in procurement. Retailer $\mathrm{G}$ cited that due to IoT data, they could provide visibility to the supplier, around two years in advance.

The most common theme in upstream integration, discussed by all retailers, was the role of IoT in inbound delivery process. Their transporters use IoT forms such as GPS telemetry to find locations, track and trace vehicles, fleet controlling, route optimization and consolidation. Some (7) spoke same thing about the international delivery tracking. Although upstream traceability was a theme discussed by four retailers, IoT (GPS tracking) has been helpful to trace and track up to first-tier supplier only[ Retailers A and I].

Three Retailers spoke of cold-chain monitoring. It was discussed that more drivers used sign-on-glass devices and hand-held devices, instead of paper-run sheets, so that everything was transmitted in realtime. IoT in receiving is a theme discussed by nine retailers. Again, they emphasised on real-time connectivity. Upstream traceability is a theme discussed by four retailers. However, IoT potential role in traceability beyond first-tier supplier was only discussed by Retailer's A and I.

In summary, all were optimistic about IoT potential in supplier integration.

\subsection{How IoT enables internal integration}

All except retailer $\mathrm{K}$ argued positively about IoT applications in their internal logistics processes. The major themes were around DC/inventory, store and HR operations.

The most common theme was the role of IoT in DCs with exception of retailer $\mathrm{E}$ and $\mathrm{F}$ who did not comment. Retailer A explained how picking was simplified efficiently ever before with the help of handheld devices. Retailer C spoke of a hand-held device, which they called MDT (mobile data terminal), that helped the forklift driver to receive, identify, and retrieve SKUs (stock keeping unit). The MDT helps forklift driver to scan and ensure the picking matched. Some retailer's location picking is mainly done through pick-to-voice, which is an Internet-connected voice recognition system. Retailer B stated about their "latest cutting-edge sortation system", which could label, scan and move cartoons to the desired location, of course using RFID (only at unit level on boxes and pallets) and the barcoding information. Security and surveillance camera which is quite energy saving and convenient, and finger print scanning for payroll purposes were some of the other themes discussed within warehousing context. The majority (except Retailer G) thought they had further potential to improve their warehousing through IoT technologies deployment. Retailer K discussed about available technologies in motion tracking using surveillance camera to detect breach of parameters and alert mechanism. Retailer $\mathrm{H}$ agreed about safety cameras with image recognition that was capable to identify inventory by scanning barcodes on goods around it. 
Except retailers A (no instore IoT) and L (e-tailer), the other ten retailers discussed how IoT driven instore technologies could help their operations. The in-store technologies vary according to the nature of the business and the industry sector. The barcoding (inner cutting, open barcodes for SKUs), POS (point of sale) devices and hand-held devices of various kinds are very common forms of in-store technologies that were used by retailers. Retailer B explained the PDA's ability to remotely scan product barcodes, allowing them to scan the price and other information, prepare price change tickets, print labels rightaway via a Wi-Fi device. Retailers C, D, H and I have smartphone apps with autonomous reporting on sales, inventory, sales target by state, including Planograms (a visual representations of each retail store's products), and alerts in real time that was shared by cross functional teams. The Retailer I explained how much individual item barcodes (closed standard) have helped them in in-store environment, especially in pricing perishables (e.g., meat, deli products) to make it fast and easy for them and the customers. Retailer $\mathrm{G}$ stated that they were currently barcode driven, but their global team was contemplating moving on the direction of RFID soon to improve accuracy of data communication to improve service level.

Retailer J in fuel retail spoke of a unique IoT application. Their underground fuel tanks have sensors fixed in them to track the stock. The sensors automatically feed the computer in the store, as well as the central operations at the head-office to monitor the fuel availability. It will send alerts in an unusual event such as theft, the fuel level that gets below a predetermined level, and the system indicates that the order needs to be dispatched and calculates when the next fuel trip is needed.

In the restaurant industry Retailer $\mathrm{E}$ believed that IoT had revolutionised the industry, for example, by using iPads to take orders. The restaurant owner further went on to explain their reconciliation system, where they registered the incoming stock using their app connected with supplies and the sales of the day to figure out the stock in hand and even a planning for the day. They have a refrigerator that is connected so that the temperature can be monitored and controlled through the phone remotely.

Retailer G has people to count the number of people who come in and out, and "sale through information" by using facial recognition, which were monitored and analysed by the head offices in real time. Retailers G, K and J had motion tracking as surveillance. Incidentally all other retailers use the simpler remote video surveillance.

Utilisation of IoT devices for identification, tracking, payroll attendance and access control was discussed as a growing trend by many interviewees of which seven were of spoke on this same theme. Out of which four employed FOBs (keyless entry devices). Retailer K explained the advantage of such application as they could digitally identify, record, monitor, track and trace each register entry in a central database along with centrally update new identities for all scanners simultaneously. Retailer K (in security and surveillance technology trade) stated that they used facial recognition (one of their own products) for access control. Retailer D spoke of how IoT had improved its internal cross functional communication.

\subsection{How IoT helps in customer integration process}

Retailers in general deliberated IoT as an application for them to connect with their customers and improved their services with lots of optimism about the future potential.

"In retail, IoT help interacting directly with the consumers. At the end, we can get the right data and communicate that effectively in terms of better service level for the customers" [Retailer G].

Customer integration generally seem to be well benefited by the current IoT disruption. The major themes were around in-store operations, understanding customers, promotions, improving the online presence, picking and despatch, deliveries, receiving and improving ratings.

IoT helps improving customer services in many forms. Except e-tailer L, all other retailers expressed the theme of IoT in store applications helping in customer interactions. Nine retailers discussed the barcoding and POS devices to improve speed and convenience for customers. Retailer I revealed that self-checkouts had made it fast and convenient to the customer. Retailers $\mathrm{C}$ and I explained that individual item barcodes on perishable products made the customer check-outs more convenient. Retailer J further mentioned about a self-checkout phone app that was piloted where, "You take your stuff, scan them and put them in the basket and walk out". Product availability due to real-time data sharing, lost sale reduction and customer retention were a theme discussed by six retailers. The way digital payment methods have had reduced the hard currency circulation was discussed by seven retailers. Retailers spoke of the role of EFTPOS machines to bring the digital currency in to the market and now the wireless version making it even more convenient. Some spoke of evolving options from a magnetic strip in bank cards to pay-wave via an electronic chip (tap and go options via NFC (near field 
communication), while others spoke of payment via mobile phones such as Apple Pay and smart watches. Retailer J mentioned an app recently introduced by a competitor that enabled the customers to pay for the fuel at the pump without going into the teller.

Understanding customer needs captured by IoT devices is a theme discussed by seven retailers. Combining POS data with reward cards to analyse demographics of the customer was brought up by many. However, Retailer G's system seems to be very advanced, where it captures video data of people walking into the stores to identify their age and their product preference. Data captured via POS systems and people counters are used together to identify customer needs and conduct a thorough forecasting. Retailer $\mathrm{G}$ revealed that they used IoT based facial recognition to demographically customise in-store advertisements of their products. Retailer K explained the scenario and confirmed that they themselves had installed facial recognition to influence customer behaviour in many retail stores.

Eight retailers spoke of exploiting IoT, of which four specifically on smartphone apps, as a means to reach the potential customers for promoting products. Another key theme discussed by six retailers is about IoT location services, specifically on smartphones helping to find nearest or specific store locations and GPS directions to get there. Retailers A, D, E, G, H and L spoke of having pervasive devices (e.g. smart phone) in customer hands that positively affected their sales. When an online order has been received, IoT ensures accurate and timely picking to enable speedy despatch discussed by four retailers who ran online operations.

Eight retailers discussed that IoT helped their customer order delivery operations. All eight retailers had their delivery operations outsourced. Out of which six customers discussed about visibility as a key benefit IoT provided to the customers. Thus, four managers stated that the customers could track their delivery by logging in to the portal. Providing good route options to deliverers is another key feature discussed by four retailers. Shorter path, optimised destination sequence, avoiding traffic chaos, and order cancellation are some advantages discussed within this theme. Five retailers spoke on the theme of IoT as a tool to receive or improve customer ratings. The communication with consumers through smartphone app though in its infancy, its capabilities are increasingly exploited and engaged for customer integration.

\subsection{Additional capabilities of IoT in supply chains}

The additional capabilities, IoT brought into supply chain operations for greater integration, were themed under visibility, auto-capture, intelligence and improved communication. The key value identified by ten retailers was improved visibility due to its pervasiveness. More importantly, six managers specifically emphasised IoT as an enabler for real-time visibility.

As retailer $\mathrm{H}$ stated, "Everyone is looking at getting real time information into the system. That's why IoT is so important for us". Seven retailers highlighted how IoT helped auto-capture data to reduce the labour requirement. Ten managers confirmed better understanding of suppliers and customers by extracting values (e.g. intelligence) from data captured by IoT. Five managers voiced positive on realtime streaming analytics, as a modern element of IoT platform. Incidentally six retailers declared that having IoT devices in their supply chain improved communication between supply chain partners.

"IoT is enabling us to communicate better with 3PLs, customers or supplies" [Retailer C].

It is evident that these additional capabilities of IoT perform beyond generic ICT on supply chain integration (SCI).

\subsection{How IoT-enabled SCI affects supply chain performance}

All twelve (12) retailers spoke positively about the outcomes of IoT currently deployed in their retail stores and their transport to improve supply chain performance. These key performance outcomes were grouped under operational performance elements like cost, quality, delivery and flexibility. Cost reduction is a key supply chain objective discussed by all participating managers. Cost savings and efficiency improvement (10), productivity (9), optimisation (8), energy saving (6), time saving (7), reduction of inventory (5) and wastage reduction (3) emerged as key themes. All twelve interviewees cited quality improvement in the name of service quality (10), accuracy (8), customer service (8), convenience (6), safety (5) and product quality (5). Nine managers voiced delivery speed (5), and timely deliveries (4) as key themes under delivery standard improvement. Ten managers cited IoT having a positive effect on flexibility of their operations. 


\subsection{How IoT-enabled supply chain performance affects firm sustainable performance}

Interviewees suggest that IoT-enabled integration improves firm performance. All agreed about its effect on triple-bottom lines of firm sustainability. All managers thought IoT deployment in supply chains affected positively their economic performance like company growth (12), sales growth (9), customer satisfaction (7), lost sales reduction (6), customer trust (5), customer retention (5), brand reputation (4), cost reduction (10), return of investments (4), and competitive edge (4). Retailer I explained that integration of technologies such as IoT and blockchain into the logistics operations could improve brand integrity, credibility and brand trust.

All twelve managers believed that IoT deployment in their supply chain operations could make their firm more environmental friendly. IoT impact on environment was diverse with declining paper use as the most discussed theme (9), followed by reduction of carbon footprints (6), electricity saving (6) and wastage minimisation (3). Reverse supply chains discussion was sparse.

Eleven managers spoke of on social sustainability theme. Under the theme, safety (7) was the key theme, with job satisfaction (5) and ease of use (4). Retailers $\mathrm{H}$ and I spoke of having these technologies in place to take a pride among staff members. Creating communities, specifically via smartphones, was a theme surfaced by four retailers.

Five retailers discussed about freeing up time to focus on more productive and innovative activities. Ten managers spoke of IoT as tool to improving their planning.

\section{Discussion}

This exploratory study aims to investigate the use of IoT in integrating the supply chain processes across retailers. The findings indicate that IoT enables supplier, internal and customer processes in Australian retail firms influencing the performance. Thematic analysis shows that all participants had clear understanding of IoT technologies in their retail operations. Despite the IoT technologies being in its early stage of adoption (Xu, He, \&Li, 2014), this qualitative study reveals that it has significant presence in select retail operations. These findings are in consistent with the earlier study by the authors, de Vass et al. (2018), where all interviewees denoted the promise of IoT as a valuable means to further integrate their supply chains. The findings of the earlier empirical study suggest that IoT capability positively affects the supplier, internal and customer integration processes that eventually improved the firm performance. The findings support several IoT related conceptual discussions, notions and arguments in the literature on its affirmative effect on SCI. For example, Ping et al. (2011)'s conceptual discussion; Yan et al. (2014)'s proposal on intelligent supply chain integration; Reaidy, Gunasekaran, and Spalanzani (2015)'s study on order fulfilment in warehouse, and more importantly de Vass et al. (2018)'s survey-based empirical study.

The study also finds that IoT-enabled SCI positively affects supply chain performance via cost, quality, delivery, and flexibility dimensions that can be attributed to IoT added capability of auto-capture, visibility (real-time), intelligence and improved communications. The exploratory findings back up the earlier scholars' arguments that emerging IoT platform is capable of improving the performance of supply chains. However, when it comes to IoT conceptualised capabilities of intelligence and automation (Constantinides et al., 2017), the potential is yet to be realised by the sampled retailers.

The thematic findings indicate that IoT-enabled supply chain performance has positive effect on firms' sustainable performance. Therefore, IoT deployment can be considered as a supply chain strategy with environmental, social, and economic goals to allow organisations to achieve its triple bottom lines of sustainability (Carter \& Rogers, 2008; Elkington, 1997).

These findings are in consistent with the findings of prior organisational capability perspectives (Huo, 2012; Rai et al., 2006), where strengthening of a higher-order integration capability has an impact on performance. This study argues that IoT deployment can enhance digitally enabled SCI, improves supply chain performance, and eventually the firm's triple bottom lines of sustainability. This study offers an understanding of IoT technologies the way operates, progresses and benefits the business operations. IoT represents a next big leap in ICT system and likely to open up exciting opportunities through the vast deployment of embedded devices (Miorandi, Sicari, De Pellegrini, \& Chlamtac, 2012).

Theoretically, this qualitative study is first of its kind so far in dealing with interviews to investigate the IoT use across retail sector. The study contributes to a small cluster of qualitative interdisciplinary exploratory body of knowledge that explains the complex role of IoT across multitude of supply chain processes. While the literature is extravagant with the potential benefits of IoT, no studies so far investigated the ground reality of IoT use. This fills the knowledge gap at theintersection of supply chain 
and IS literature by establishing the fact that IoT-enabled SCI improves firm performance. Practically, the qualitative findings from the retailers provide interesting insights to practitioners in the field of supply chain and IS. The thematic findings offer insights and intelligence that arise from the investment of IoT where managers, industry associations and policy makers are highly benefited. Managers need to understand the power of IoT while deciding on any investment on logistics operations. Policy makers' understanding of IoT technologies will influence a sound public investment of these emerging pervasive technologies.

\section{Conclusion}

The effect of IoT on SCI and firm performance is quantitatively validated in a prior framework-based study. However, descriptive evidences to explain the role of IoT in current supply chain context is vital to better understand the phenomenon. The findings of this semi-structured interview-based qualitative study of 12 retailers provided detailed support for how IoT-enabled SCI influences supply chain and firm performance. The confirmation that IoT not only integrates internal logistics but also external partner (i.e. suppliers and customers) integration that influences positively on cost, quality, delivery, and flexibility of the entire supply chain to improve firm's sustainable performance with financial, social and environmental outcomes. The study opens up status quo of IoT technologies in Australian retail supply chains, and how they are deployed to strengthen SCI. IoT additional capabilities of supporting visibility, auto-capture, intelligence, and inter-and intra-firm communication are perceived to be accountable for this performance improvement in the current supply chain context.

There are some limitations in this study. First, the drivers, constraints and enablers of IoT adoption were given a little consideration. Although twelve retail cases are adequate enough to consolidate a phenomenon, the current findings are generalizable within the cases only. The equitable cases from each retail group are proposed in a study ahead to get a more evidence for generalisation. Second, even though IoT is discussed for its potential to track and trace entities throughout the supply chain (Kiritsis, 2011; Lianguang, 2014), the study explored its ability only from the unilateral focal retailers perspective. Therefore, exploring IoT capability from other firms in the supply chain (e.g. distributors, transporters) will reveal its most touted capability of sensing, capturing, and communicating data with others. A survey study grounded on qualitative findings will be appropriate for this purpose.

\section{References}

ABS_ANZSIC. 2013. Australian and New Zealand Standard Industrial Classification (ANZSIC), 2006 (Revision 2.0). Retrieved from http:// www.abs.gov.au/ausstats/abs@.nsf/Latestproducts/42D687FA072ACCC6CA257B950 0133B9B?opendocument

Alfalla-Luque, R., Medina-Lopez, C., \& Dey, P. K. 2013. 'Supply chain integration framework using literature review'. Production Planning \& Control, 24(8-9), 800-817.

Atzori, L., Iera, A., \& Morabito, G. (2010). 'The Internet of Things: A survey". Computer Networks, 54(15), 2787-2805.

Balaji, M., \& Roy, S. K. 2017. 'Value co-creation with Internet of things technology in the retail industry'. J ournal of Marketing Management, 33(1-2), 7-31.

Ben-Daya, M., Hassini, E., \& Bahroun, Z. 2017. 'Internet of things and supply chain management: a literature review'. International J ournal of Production Research, 1-24.

Borgia, E. (2014). 'The Internet of Things vision: Key features, applications and open issues'. Computer Communications, 54, 1-31.

Carter, C. R., \& Rogers, D. S. 2008. 'A framework of sustainable supply chain management: moving toward new theory'. International J ournal of Physical Distribution \& Logistics Management, 38(5), 360-387.

Constantinides, E., Kahlert, M., \& de Vries, S. A. 2017. 'The relevance of technological autonomy in the acceptance of IoT services in retail'. Paper presented at the 2nd International Conference on Internet of Things, Data and Cloud Computing, ICC 2017.

Creswell, JW \& Poth, CN 2017, Qualitative inquiry and research design: Choosing among five approaches, Thousand Oaks: SAGE Publications.

Cui, Y. 2015. 'Improving Supply Chain Resilience with Employment of IoT'. Paper presented at the International Conference on Multidisciplinary Social Networks Research.

de Vass, T., Shee, H., \& Miah, S. J. 2018. 'The effect of "Internet of Things" on supply chain integration and performance: An organisational capability perspective'. Australasian Journal of Information Systems, 22.

Dweekat, A. J ., \& Park, J . 2016. Internet of Things-Enabled Supply Chain Performance Measurement 
Model. Paper presented at the Industrial Engineering, Management Science and Application (ICIMSA), 2016 International Conference on.

Elkington, J . (1997). Cannibals with forks. The triple bottom line of 21st century.

Haddud, A., DeSouza, A., Khare, A., \& Lee, H. 2017. 'Examining potential benefits and challenges associated with the Internet of Things integration in supply chains'. J ournal of Manufacturing Technology Management, 28(8), 1055-1085.

Hofmann, E., \& Rüsch, M. 2017. 'Industry 4.0 and the current status as well as future prospects on logistics'. Computers in Industry, 89, 23-34.

Huo, B. 2012. 'The impact of supply chain integration on company performance: an organizational capability perspective'. Supply Chain Management: An International J ournal, 17(6), 596-610.

Kim, H. J . 2017. 'Information technology and firm performance: the role of supply chain integration'. Operations Management Research, 10(1), 1-9.

Kiritsis, D. 2011. 'Closed-loop PLM for intelligent products in the era of the Internet of things'. Computer-Aided Design, 43(5), 479-501.

Li, G., Yang, H., Sun, L., \& Sohal, A. S. 2009. 'The impact of IT implementation on supply chain integration and performance'. International J ournal of Production Economics, 120(1), 125-138.

Lianguang, M. 2014. Study on supply-chain of modern agricultural products based on iot in order to guarantee the quality and safety. Advance J ournal of Food Science and Technology, 6(4).

Liu, W., \& Gao, Z. 2014. 'Study on IOT based architecture of logistics service supply chain'. International J ournal of Grid and Distributed Computing, 7(1), 169-178.

Liu, X., \& Sun, Y. 2011. 'Information Flow Management of Vendor-Managed Inventory System in Automobile Parts Inbound Logistics Based on Internet of Things'. J ournal of Software, 6(7), 1374-1380.

Majeed, A. A., \& Rupasinghe, T. D. 2017. 'Internet of things (IoT) embedded future supply chains for industry 4.0: An assessment from an ERP-based fashion apparel and footwear industry'. International J ournal of Supply Chain Management, 6(1), 25-40.

Miorandi, D., Sicari, S., De Pellegrini, F., \& Chlamtac, I. 2012. 'Internet of things: Vision, applications and research challenges'. Ad Hoc Networks, 10(7), 1497-1516.

Mishra, D., Gunasekaran, A., Childe, S. J., Papadopoulos, T., Dubey, R., \& Wamba, S. F. 2016. 'Vision, applications and future challenges of Internet of Things'. Industrial Management \& Data Systems, 116(7), 1331-1355.

Ping, L., Liu, Q., Zhou, Z., \&Wang, H. 2011. Agile supply chain management over the internet of things. Paper presented at the Management and Service Science (MASS), 2011 International Conference on 2011 Aug 12.

Rai, A., Patnayakuni, R., \& Seth, N. 2006. 'Firm performance impacts of digitally enabled supply chain integration capabilities'. MIS Quarterly, 30(2), 225-246.

Reaidy, P.J ., Gunasekaran, A., \& Spalanzani, A. 2015. 'Bottom-up approach based on Internet of Things for order fulfillment in a collaborative warehousing environment'. International J ournal of Production Economics, 159(1), 29-40.

Whitmore, A., Agarwal, A., \& Da Xu, L. 2014. 'The Internet of Things-A survey of topics and trends'. Information Systems Frontiers, 17(2), 261-274.

Wieland, A., \& Marcus Wallenburg, C. 2012. 'Dealing with supply chain risks'. International J ournal of Physical Distribution \& Logistics Management, 42(10), 887-905.

$\mathrm{Xu}, \mathrm{L}$. D., He, W., \&Li, S. 2014. 'Internet of Things in industries: A survey'. Industrial Informatics, IEEE Transactions on, 10(4), 2233-2243.

Yan, J., Xin, S., Liu, Q., Xu, W., Yang, L., Fan, L., ... Wang, Q. 2014. 'Intelligent supply chain integration and management based on Cloud of Things'. International J ournal of Distributed Sensor Networks, 10(3), 1-15.

$\mathrm{Yu}, \mathrm{W}$. 2015. 'The effect of IT-enabled supply chain integration on performance'. Production Planning \&Control, 26(12), 945-957.

Copyright: (C) 2018 authors. This is an open-access article distributed under the terms of the Creative Commons Attribution-NonCommercial 3.0 Australia License, which permits non-commercial use, distribution, and reproduction in any medium, provided the original author and ACIS are credited. 